\title{
SURVEY OF THE VASCULAR FLORA OF THE BOEHLER SEEPS AND SANDHILLS PRESERVE
}

\author{
Submitted to the Department of Botany of Oklahoma State University \\ in partial fulfillment of the requirements for the Degree of Doctor of Philosophy \\ July 1997
}

\section{Linda Gatti Clark \\ E-mail: gatti.clark@gmail.com}

Located in Atoka County of southcentral Oklahoma, The Nature

Conservancy's Boehler Seeps and Sandhills

Preserve comprises sandhills, acidic hillside seeps, marshes, intermittent and permanent streams, and shallow lakes. The sandhills are the site of the highest quality, old-growth vegetation of the Western Gulf Coastal Plains (S. Orzell, pers. comm. to Ian Butler). The flora is a unique assemblage of plants that is present nowhere else in the state and considered globally rare. Approximately 400 species are believed to be present (Jones 1993). More than 20 rare species have been reported to occur in the area, including some that are globally rare (Oklahoma Natural Heritage Inventory 1997). Eriocaulon kornickianum, for example, is designated G2 and $\mathrm{S1}$. Other rare species in the preserve include Penstemon murrayanus (G4, S1S3), Polygonella americana (G5, S1S2), and Paronychia drummondii (G4G5, S1S2).

Prior to this study, our knowledge of the vascular plant species in the preserve was incomplete. Although several partial lists of its flora had been compiled, a systematic survey of the area to inventory all of the plants had not been conducted. Such information is essential for understanding the ecology of the site and making decisions about its management. This study was undertaken to provide this information. Specific objectives were to: (1) compile a list of the terrestrial and aquatic vascular plant species present and (2) prepare a set of herbarium specimens to document the preserve's flora. This note summarizes my findings and provides a reference to the information compiled in Gatti Clark (1997).

\section{BOEHLER SEEPS AND SANDHILLS PRESERVE}

The preserve is a 235 ha site located in southern Atoka County, Oklahoma, approximately 11 miles north of Boswell (S25 \& 26, T4S, R13W; Boswell NW Quad). It comprises two tracts bisected by a paved country road and is located in the watersheds of Muddy Boggy and Clear Boggy Creeks. Situated in the Dissected Coastal Plain Geomorphic Province and Western Coastal Plain Land Resource Area (Johnson et al. 1979, USDA Natural Resources Conservation Service 1992), its underlying strata are Cretaceous in age. Also underlying the preserve is the Antlers Sandstone Aquifer that is composed primarily of non-marine sand and clay, and marine limestone and clay up to $915 \mathrm{~m}$ thick and is saturated with water that has a moderate to high mineral content (Johnson et al. 1979). The water table is generally within 1-1.3 m of the surface, with seeps occurring where it reaches the surface (Jones 1993, pers. comm.). Soil series of the site are the Bernow-Romia complex, 8-12\% slopes; Boggy fine sandy loam; and Larue loamy fine sand, $0-8 \%$ slopes (Shingleton and Watterson 1979). All are susceptible to erosion by both water and wind. Precipitation in the area of the preserve

Clark, L. G. 
occurs primarily in the spring and summer, and averages $119 \mathrm{~cm}$ per year (Ruffner 1980). The average growing season is 255 days; mean maximum annual temperature is $24.5^{\circ} \mathrm{C}$ and mean minimum is $11^{\circ} \mathrm{C}$; the average number of days below $0^{\circ} \mathrm{C}$ is 52 (Ruffner 1980).

Vegetation of the area is Oak-Hickory Forest (Duck and Fletcher 1943) with several communities present. Quercus stellata, Carya texana, and Sideroxylon lanuginosum spp. Lanuginosum (=Bumelia lanuginose) dominate and form an almost continuous canopy in the drier areas. Typically a thick leaf layer is present on the ground, and understory vegetation is sparse. Juniperus virginiana and Pinus echinata, while not dominant, can be found scattered throughout the preserve. Common woody understory species are Nyssa sylvatica, $V$ accinium arboreum, Berchemia scandens, and $V$ itis rotundifolia. Herbaceous understory taxa include Galium arkansanum, G. obtusum, Tephrosia virginia, Carex spp., Cyperus spp., and Juncus spp. Scattered throughout the preserve and most conspicuous are glades dominated by Aristida desmantha and Selaginella rupestris. Other glades are present and are dominated by various grasses, such as Panicum spp., mosses, and forbs, such as Gaillardia aestivalis and Hieracium longipilum. At the glade edges, trees other than the forest dominants are encountered, in particular Quercus incana. Its saplings are occasionally found in the centers of the glades.

Hassel and Boehler Lakes are small, shallow bodies of water maintained by beaver dams. Both have dense stands of emergent and floating-leaved species at their edges and open water in their centers. Dominant taxa include Typha angustifolia, Nuphar lutea, and Nymphaea odorata. Often quite abundant, free-floating species are Azolla caroliniana and Utricularia biflora. Two types of seeps are present in the preserve. One has water percolating slowly to the surface and accumulating in one area because of the topography. The ground surface has a spongy feel because of the thick carpet of vegetation, primarily mosses; Sphagnum lescurii and Polytrichum commune in particular dominate. Occupying natural drainage ways, the second type is characterized by water flowing away from the seepage point. Ferns, sedges, and rushes typically are in abundance along these watercourses. Between the lakes and the seeps are marshes dominated by Osmunda regalis, O. cinnamonea, Cephalanthus occidentalis, Scirpus spp., Rhynchospora spp., and Cyperus spp. Associated with Boehler Lake are rather deep drainages that resemble sloughs. They have less vegetation and are often banked by large trees such as Quercus falcata, Q. nigra, and Q. phellos.

\section{METHOD OF SURVEY}

A systematic collection of the terrestrial and aquatic vascular plants occurring in the preserve was conducted during the 1994 and 1995 growing seasons. The area was divided into three survey units using roads and fences as boundaries. Each unit was traversed on foot several times during the growing season. Plants were collected in both the flowering and fruiting stages, and prepared using standard herbarium techniques (Radford et al. 1974). One set of 440 voucher herbarium specimens (Appendix H) was prepared and deposited in the Oklahoma State University Herbarium (OKLA). Specimens previously collected by Conservancy personnel were identified and included in the inventory (Gatti Clark 1997, Appendices I and J). Identification was accomplished using the resources of the herbarium. Nomenclature used was based primarily on that of Waterfall (1969), Correll and Johnston (1979), and Gray's Manual of Botany (Fernald 1950). Common names were taken from Correll and Johnston (1979) and Taylor and Taylor (1994). 


\section{FLORA OF THE PRESERVE}

Three hundred forty-five species in 225 genera and 84 families were encountered in this survey or by previous workers (Gatti Clark 1997, Appendices H, I, and J). Three families, Asteraceae (56 taxa), Poaceae (41 taxa), and Cyperaceae (35 taxa), composed $38 \%$ of the preserve's vascular flora. Other large families were the Fabaceae (21 taxa), Apiaceae (11 taxa), Lamiaceae (10 taxa), and Scrophulariaceae (10 taxa). The largest genera present were Carex, represented by 17 species, and Panicum, represented by 12 species.

Species designated by the U.S. Fish and Wildlife Service (1996) as endangered, threatened, or candidate (formally category 1) were not encountered. Species ranked by the ONHI (1997) as S1 or S2 and present in the preserve included the previously mentioned Eriocaulon kornickianum (G2, S1), Penstemon murrayanus (G4, S1S3), Polygonella americana (G5, S1S2), and Paronychia drummondii (G4G5, S1S2). Other rare species are listed in the table.

Although demonstrably secure globally and ranked G4 or G5 by ONHI, several species of interest were found in the preserve. The insectivorous Drosera brevifolia and the mycotrophic/parasitic Monotropa bypopithys were encountered. Lycopodiella appressa (=Lycopodium appressum) is reported for the site but was not seen in this study or in collections of The Nature Conservancy personnel (L. K. Magrath, pers. comm.).

With 22 rare taxa reported for the site, monitoring of the Bluejack Oak sandhills and seep communities should continue. The communities and its assemblage of plants at Boehler Seeps and Sandhills Preserve are rare within the state and deserve continued study.

\section{LITERATURE CITED}

Correll, D. S. and M. S. Johnston. 1970. Manual of the V ascular Plants of Texas. Texas Research Foundation, Renner, TX.

Duck, L. G. and J. B. Fletcher. 1943. A Game Type Map of Oklahoma. State of Oklahoma Game and Fish Department, Division of Wildlife Restoration. Oklahoma Biological Survey, Norman, OK.

Fernald, M. L. 1950. Gray's Manual of Botany. $8^{\text {th }}$ ed. American Book Company, New York.

Gatti Clark, L. C. 1997. Floristic and Biosystematic Investigations in Plant Taxonomy. Ph.D. dissertation. Oklahoma State University, Stillwater, OK.

Jones, N. 1993. A baseline study of the water quality, vegetative gradients, and hydrology of Boehler Seeps and Sandhills Preserve. Report. The Nature Conservancy, Tulsa, OK.

Johnston, K. S., C. C. Branson, N. M. Curtis, Jr., W. E. Ham, W. E. Harrison, M. V. Marcher, and J. F. Roberts. 1979. Geology and Earth Resources of Oklahoma. Educational Publication 1. Oklahoma Geological Survey, Norman, OK.

Oklahoma Natural Heritage Inventory. 1997. Short Working List of Rare Plants. Version of 22 January 1997. Oklahoma Biological Survey, Norman, OK.

Radford, A. E., W. C. Dickison, J. R. Massey, and C. R. Bell. 1974. Vascular Plant Systematics. Harper and Row, New York.

Ruffner, J. A. 1980. Climate of the United States: National Oceanic and Atmospheric Administration Narrative Summaries, Tables, and Maps for Each State with Overview of State Climatologist Programs. $2^{\text {nd }}$ ed.

Volume 2. Gale Research Company, Detroit, MI. 
Shingleton, L. C. and A. Watterson, Jr. 1979. Soil Survey of Atoka County, Oklahoma. USDA Soil Conservation Service, Stillwater, OK.

Taylor, R. J. and C. E. S. Taylor. 1994. An Annotated List of the Ferns, Fern Allies, Gymnosperms, and Flowering Plants of Oklahoma. $3^{\text {rd }}$ ed. Published by authors, Durant, OK.

USDA Natural Resources Conservation Service. 1992. Map of Major Land
Resource Areas of Oklahoma. Stillwater, OK.

US Fish and Wildlife Service. 1996.

Endangered and Threatened Wildlife and Plants. October 31, 1996. 50 CFR 17.11-17.12. Washington, D. C.

Waterfall, U. T. 1969. Keys to the Flora of Oklahoma. $4^{\text {th }}$ ed. Published by author, Stillwater, OK.

Clark, L. G. 
Table Taxa of Boehler Seeps and Sandhills Preserve that are ranked as rare by the Oklahoma Natural Heritage Inventory (ONHI)

\begin{tabular}{llcc}
\hline & & \multicolumn{2}{c}{ ONHI Rankings* } \\
\cline { 3 - 4 } \multicolumn{1}{c}{ Scientific Name } & \multicolumn{1}{c}{ Common Name } & Global & State \\
\hline Agalinis tenuifolia (Vahl.) Raf. & slender leaved agalinis & G5 & S2S3 \\
Aristolochia reticulata Jacq. & netleaved snakeroot & G4 & S2 \\
Azolla caroliniana Willd. & mosquito fern & G5 & S2 \\
Brasenia schreberi J. F. Gmel. & water-shield & G5 & S1 \\
Carex hyalina Boott & whitesheath sedge & G5 & S1 \\
Carex swanii (Fernald) Mack. & swan sedge & G5 & S1 \\
Carya myristiciformis (Michx. f.) Nutt. & nutmeg hickory & G5 & S2S3 \\
Drosera brevifolia Pursh & sundew & G5 & S2S3 \\
Dulichium arundinaceum (L.) Britton & threeway sedge & G5 & S1 \\
Eriocaulon kornickianum van Heurch and Müll.Arg. & small pipewort & G2 & S1 \\
Galium arkansanum A. Gray & Arkansas bedstraw & G5 & S1S2 \\
Houstonia micrantha (Shinners) Terrell & bluet & G4G5 & S1S2 \\
(=Hedyotis australis W.H. Lewis \& D.M. Moore) & & & \\
Iris virginica L. & southern blue flag & G5 & S2? \\
Monotropa hypopithys L. & pinesap & G5 & S1 \\
Paronychia drummondii Torr. \& A. Gray & Drummond's nailwort & G4G5 & S1S2 \\
Penstemon murrayanus Hook. & cupleaf beardtongue & G4 & S1S3 \\
Platanthera flava (L.) Lindl. & pale green orchid & G4 & S1 \\
Polygonella americana (Fisch. \& C.A. Mey.) Small & southern jointweed & G5 & S1S2 \\
Quercus incana Bartram & bluejack oak & G5 & S1S2 \\
Rhynchospora caduca Elliott & anglestem beakrush & G5 & S1 \\
Saccharum giganteum (Walter) Pers. & giant plumegrass & G5 & S1S2 \\
(=Erianthus giganteus (Walter) P. Beauv.) & & & \\
Sacciolepis striata (L.) Nash & American cupscale & G5 & S2 \\
\hline OOnHIG & & &
\end{tabular}

${ }^{*} \mathrm{ONHI}$ Global Rankings:

G2 - Imperiled globally because of its rarity ( 6 to 20 occurrences or few remaining individuals or acres) or because of other factors demonstrably making it vulnerable to extinction throughout its range.

G4 - Apparently secure globally, though it may be quite rare in parts of its range, especially at the periphery.

G5 - Demonstrably secure globally though it may be quite rare in parts of its range, especially at the periphery.

S1 - Critically imperiled in Oklahoma because of extreme rarity ( 5 or fewer occurrences or very few remaining individuals or acres) or because of some factor of its biology making it especially vulnerable to extinction.

S2 - Imperiled in Oklahoma because of extreme rarity (6 to 20 occurrences or few remaining individuals or acres) or because of other factors making it very vulnerable to extinction throughout its range.

S3 - Rare and local in Oklahoma (thought it may be abundant at some of its locations); in the range of 21-100 occurrences.

Clark, L. G. 


\section{APPENDIX \\ Vascular Plant Collections from Boehler Seeps and Sandhills Preserve arranged by family. Collections of L. C. Gatti Clark and The Nature Conservancy personnel.}

[Ed. Notes: All plants are collected by L. Gatti Clark, unless indicated by an asterisk * for the Nature Conservancy or a tilde $\sim$ for plants collected by both. Nomenclature has been updated using the PLANTS Database (plants.usda.gov/plants).]

\section{FERN ALLIES}

Selaginellaceae - Spikemoss Family

Selaginella rupestris (L.) Spring

rock spikemoss

\section{FERNS}

Azollaceae - Azolla Family

Azolla caroliniana Willd.

mosquito fern

Dryopteridaceae - Wood Fern Family

Onoclea sensibilis L.

sensitive fern

Woodsia obtusa (Spreng.) Torr.

blunt-lobed cliff fern

Ophioglossaceae - Adder's-Tongue Family

Botrychium virginianum (L.) Sw.

rattlesnake fern

Osmundaceae - Royal Fern Family

Osmunda cinnamomea L.

cinnamon fern

$\sim$ Osmunda regalis $L$. var. spectabilis

(Willd.) A. Gray

royal fern

GYMNOSPERMS

Cupressaceae - Cypress Family

Juniperus virginiana L.

eastern redcedar

Pinaceae - Pine Family

$\sim$ Pinus echinata Mill.

shortleaf pine

ANGIOSPERMS

Liliopsida - Monocots

Alismataceae - Water Plantain Family

Alisma subcordatum Raf.

Echinodorus tenellus (Mart.ex Schult. f.) Buchenau

Sagittaria latifolia Willd.

water plaintain

lanceleaf burweed

wapato, duck potato

Commelinaceae - Spiderwort Family

Commelina erecta L.

erect day flower

Tradescantia ohiensis Raf.

Ohio spiderwort

Clark, L.G. 
Cyperaceae - Sedge Family

Carex bicknellii Britton

Carex blanda Dewey

Carex cherokeensis Schwein.

* Carex complanata Torr. \& Hook. Carex crinita Lam.

$\sim$ Carex digitalis Willd.

* Carex frankii Kunth Carex granularis Muhl. ex Willd.

Carex gravida L.H. Bailey

Carex hyalina Boott

Carex lupulina Muhl. Ex Willd.

* Carex muehlenbergii Schkuhr ex Willd.

Carex normalis Mack.

Carex retroflexa Muhl. ex Willd.

* Carex squarrosa L.

Carex swanii (Fernald) Mack.

Carex vulpinoidea Michx.

$\sim$ Cyperus echinatus (L.) Alph. Wood

(=C. ovularis (Michx.) Torr.)

Cyperus retroflexus Buckley

(=C. uniflorus Torr. \& Hook., non Thunb.

* Cyperus strigosus L.

Cyperus virens Michx.

Dulichium arundinaceum (L.) Britton

Eleocharis acicularis var. acicularis (L.) Roem. \& Schult.

(=E. acicularis (L.) Roem. \& Schult. var. gracilescens)

$\sim$ Eleocharis compressa Sull.

Eleocharis engelmannii Steud.

$\sim$ Eleocharis lanceolata Fernald

(=E. obtusa (Willd.) Schultes var. lanceolata (Fernald) Gilly

Eleocharis parvula (Roem. \& Schult.) Link ex Bluff, Nees.

\& Schauer (=E. parvula (Roem. \& Schult.) Link var.

anachaeta (Torr.) Svens.

Eleocharis tenuis (Willd.) Schult.

var. verrucosa (Svens.) Svens.

Isolepis carinata Hook. \& Arn. ex Torr.

(=Scirpus koilolepis (Steud.) Gleason

* Lipocarpha aristulata (Coville) G. Tucker

(=Hemicarpha aristulata (Coville) Smyth

Rhynchospora caduca Elliott

$\sim$ Rhynchospora capitellata (Michx.) Vahl

Rhynchospora glomerata (L.) Vahl

Scleria ciliata Michx.

Scleria triglomerata Michx.
Bicknell's sedge

loose flowered sedge

Cherokee sedge

sedge

fringed sedge

sedge

Frank's sedge

meadow sedge

heavy sedge

whitesheath sedge

hop sedge

Muhlenberg's sedge

sedge

reflexed sedge

sedge

swan sedge

fox sedge

globe flatsedge

one-flower flatsedge

false nutgrass

green flatsedge

threeway sedge

needle spikesedge

flatstem spikesedge

Engleman's spikesedge

blunt spikesedge

dwarf spikesedge

slender spikesedge

bulrush

hemicarpa

anglestem beakrush

false bogrush

clustered beakrush

fringed nutrush

whip nutrush

Clark, L. G. 
Eriocaulaceae - Pipewort Family

Eriocaulon kornickianum van Heurch \& Müll. Arg. small pipewort

Iridaceae - Iris Family

Iris virginica L.

Sisyrinchium angustifolium Mill.

southern blue flag

blue-eyed grass

Juncaceae - Rush Family

Juncus acuminatus Michx.

jointed rush

$\sim$ Juncus coriaceus Mack. Juncus effusus $L$.

Juncus marginatus Rostk.

leathery rush

bog rush

$\sim$ Juncus scirpoides Lam.

grassleaf rush

needlepod rush

Juncus tenuis Willd.

Luzula bulbosa (Alph. Wood) Smyth \& Smyth

tender rush

bulb woodrush

Lemnaceae - Duckweed Family

Spirodela polyrrhiza (L.) Schleid.

duck meat

Liliaceae - Lily Family

$\sim$ Allium canadense $\mathrm{L}$.

Hypoxis hirsuta (L.) Coville

wild onion

yellow stargrass

Orchidaceae - Orchid Family

* Platanthera flava (L.) Lindl.

pale green orchid

Poaceae - Grass Family

Agrostis perennans (Walter) Tuck.

* Andropogon gerardii Vitman Andropogon ternarius Michx.

Aira elegans Willd. ex Kunth

Aristida desmantha Trin. \& Rupr.

Bouteloua hirsuta Lag.

Bromus arvensis L. (=B. japonicus Thunb.)

Bromus catharticus Vahl

* Bromus hordeaceus $\mathrm{L}$.

$\sim$ Cenchrus spinifex Cav. (=C. incertus M. A. Curtis)

$\sim$ Chasmanthium latifolium (Michx.) Yates

$\sim$ Chasmanthium sessiliflorum (Poir.) Yates (=C. laxum (L.) Yates spp. sessiliflorum (Poir.) L. Clark)

Danthonia spicata (L.) P. Beauv. ex Roem. \& Schult.

$\sim$ Dichanthelium acuminatum (Sw.) Gould \& C.A. Clark var. fasciculatum (Torr.) Freckmann (=Panicum lanuginosum Eliott, non Bosc ex Spreng.)

Dichanthelium boscii (Poir.) Gould \& C.A. Clark (=Panicum boscii Poir.)

autumn bentgrass

big bluestem

splitbeard bluestem

annual silver hairgrass

curly threeawn

hairy grama

Japanese brome

rescue grass

soft chess

sandbur

inland seaoats

spike-inland seaoats

poverty oatgrass

wooly panicum

Bosc panicum

Clark, L.G. 
Dichanthelium depauperatum (Muhl.) Gould (=Panicum depauperatum Muhl.)

Dichanthelium dichotomum (L.) Gould var. dichotomum (=Panicum dichotomum L.)

Dichanthelium linearifolium (Scribn. ex Nash) Gould (=Panicum linearifolium Scribn.)

Dichanthelium oligosanthes (Schult.) Gould var. oligosanthes (=Panicum oligosanthes Schult.)

Dichanthelium ravenelli (Scribn. \& Merr.) Gould (=Panicum ravenelii Scribn. \& Merr.)

Dichanthelium sphaerocarpon (Elliott) Gould var. sphaerocarpon (=Panicum sphaerocarpon Elliott)

$\sim$ Elymus virginicus L.

* Eragrostis capillaris (L.) Nees

* Eragrostis hirsuta (Michx.) Nees

Eragrostis secundiflora J. Presl

Eragrostis spectabilis (Pursh) Steud.

Gymnopogon ambiguus (Michx.) Britton, Sterns \& Poggenb. Leersia oryzoides (L.) Sw.

$\sim$ Panicum anceps Michx. Panicum dichotomiflorum Michx.

* Paspalum laeve Michx.

Paspalum setaceum Michx.

* Saccharum giganteum (Walter) Pers. (=Erianthus giganteus (Walter) P. Beauv.)

Sacciolepis striata (L.) Nash

Setaria parviflora (Poir.) Kerguélen

(=S. geniculata (Willd.) P. Beauv., nom. illeg.)

Sorghum halepense (L.) Pers.

Sphenopholis obtusata (Michx.) Scribn.

Steinchisma hians (Elliott) Nash (=Panicum hians Elliott)

$\sim$ Tridens flavus (L.) Hitchc.

Vulpia octoflora (Walter) Rydb.

Zizaniopsis miliacea (Michx.) Döll. \& Asch.

Potemogetonaceae - Pondweed Family

Potamogeton pulcher Tuck.

Smilacaceae - Catbriar Family

Smilax bona-nox L.

Smilax rotundifolia $\mathrm{L}$.

Typhaceae - Cattail Family

Typha angustifolia L. slimleaf panicum

forked panicum

slimleaf panicum

small panicgrass

panicum

leafy panicum

Virginia wildrye

lacegrass

bigtop lovegrass

red lovegrass

purple lovegrass

broadleaf skeletongrass

Swartz cutgrass

beaked panicum

fall panicum

field paspalum

thin paspalum

giant plumegrass

American cupscale

knotroot bristlegrass

Johnsongrass

prairie wedgescale

gaping panicum

purpletop

sixweeks fescue

southern wildrice

spotted pondweed

greenbrier

common greenbrier

narrow-leaved cattail

Clark, L. G. 
Magnoliopsida - Dicots

Acanthaceae - Acanthus Family

Ruellia humilis Nutt.

fringed leaf ruellia

Amaranthaceae - Amaranth Family

Froelichia floridana (Nutt.) Moq.

snake cotton

Anacardiaceae - Sumac Family

Rhus aromatica Aiton

Rhus copallinum L. (=R. copallina L., orth. var.)

Toxicodendron radicans (L.) Kuntze

lemon sumac

winged sumac

poison ivy

Apiaceae - Carrot Family

* Chaerophyllum tainturieri Hook.

hairy fruit wild chervil

$\sim$ Daucus pusillus Michx. Eryngium prostratum Nutt. ex DC.

* Hydrocotyle verticillata Thunb.

$\sim$ Ptilimnium capillaceum (Michx.) Raf.

$\sim$ Sanicula canadensis L.

Sanicula odorata (Raf.) K.M. Pryer \& L.R. Phillippe

(=S. gregaria E.P. Bicknell)

Spermolepis divaricata (Walter) Raf. ex Ser.

Spermolepis echinata (Nutt. ex DC.) A. Heller

Spermolepis inermis (Nutt. ex DC.) Mathias \& Constance

Torilis arvensis (Huds.) Link

southwestern carrot

creeping eryngo

whorled pennywort

threadleaf mockbishopweed

black snakeroot

cluster snakeroot

forked scaleseed

bristly scaleseed

spreading scaleseed

hedge parsley

Apocynaceae - Dogbane Family

Apocynum cannabinum $\mathrm{L}$.

Indianhemp

Aquifoliaceae - Holly Family

Ilex decidua Walter

deciduous holly

Aristolochiaceae - Birthwort Family

$\sim$ Aristolochia reticulata Jacq.

netleaved snakeroot

Asclepiadaceae - Milkweed Family

Asclepias tuberosa L.

Asclepias verticillata L.

Asclepias viridis Walter

Matelea biflora (Raf.) Woodson

butterfly milkweed

whorled milkweed

green milkweed

twoflower milkvine

Asteraceae - Sunflower Family

* Achillea millefolium L.

yarrow

* Ambrosia artemisiifolia L.

Ambrosia bidentata Michx.

* Ambrosia trifida L.

Antennaria parlinii Fernald

common ragweed

lanceleaf ragweed

giant ragweed

plainleaf pussytoes

Clark, L.G. 
Astranthium integrifolium (Michx.) Nutt.

* Bidens aristosa (Michx.) Britton Centaurea americana Nutt.

* Chaetopappa asteroides Nutt. ex DC.

$\sim$ Chrysopsis pilosa Nutt.

* Cirsium altissimum (L.) Hill Cirsium horridulum Michx.

* Conoclinium coelestinum (L.) DC. (=Eupatorium coelestinum L.)

$\sim$ Conyza canadensis (L.) Cronquist

$\sim$ Coreopsis grandiflora Hogg ex Sweet

$\sim$ Croptilon divaricatum (Nutt.) Raf.

(=Haplopappus divaricatus (Nutt.) A. Gray)

$\sim$ Echinacea pallida (Nutt.) Nutt.

$\sim$ Elephantopus carolinianus Raeusch.

* Erechtites hieracifolia (L.) Raf. ex DC.

$\sim$ Erigeron strigosus Muhl. ex Willd.

* Eupatorium perfoliatum L. Evax prolifera Nutt. ex DC.

Evax verna Raf. var. verna (=E. multicaulis DC.) Gaillardia aestivalis (Walter) $\mathrm{H}$. Rock

Gamochaeta purpurea (L.) Cabrera (=Gnaphalium purpureum L.)

* Helenium amarum (Raf.) H. Rock

* Helianthus angustifolius $\mathrm{L}$.

$\sim$ Helianthus hirsutus Raf. Heterotheca villosa (Pursh) Shinners var. villosa (=Chrysopsis villosa (Pursh.) Nutt. ex DC.)

$\sim$ Hieracium gronovii $\mathrm{L}$. Hieracium longipilum Torr. Hymenopappus scabiosaeus L'Her.

$\sim$ Krigia cespitosa (Raf.) K. L. Chambers

$\sim$ Krigia dandelion (L.) Nutt. Krigia virginica (L.) Willd. Lactuca canadensis L.

* Lactuca sativa L. Liatris aspera Michx.

* Liatris elegans (Walter) Michx. Liatris squarrosa (L.) Michx.

* Mikania scandens (L.) Willd. Packera obovata (Muhl. ex Willd.) W.A. Weber \& A. Love (=Senecio obovatus Muhl. ex Willd. var. rotundus Britton)

* Pluchea camphorata (L.) DC. Pseudognaphalium obtusifolium (L.) Hilliard \& B.L. Burtt ssp. obtusifolium (=Gnaphalium obtusifolium L.)

Pyrrhopappus carolinianus (Walter) DC.

* Rudbeckia grandiflora (D. Don) J.F. Gmel. ex DC.

Rudbeckia hirta L. western daisy tickseed sunflower American basket flower least daisy softhair golden aster tall thistle bull thistle blue boneset

horseweed bigflowered tickseed scratch daisy

pale coneflower elephant's foot fireweed daisy fleabane boneset rabbit's tobacco rabbit's tobacco prairie gaillardia purple cudweed sneezeweed narrow-leaf sunflower hairy sunflower roughhair golden aster

hawkweed longbeard hawkweed old plainsman common dwarf dandelion potato dandelion dwarf dandelion wild lettuce prickly lettuce tall gayfeather beautiful gayfeather gayfeather climbing hempweed roundleaf groundsel

camphorweed

sweet everlasting

false dandelion Mexican hat blackeyed Susan

Clark, L. G. 
Solidago canadensis $\mathrm{L}$.

Solidago missouriensis Nutt.

* Solidago odora Aiton

* Solidago rugosa Mill.

Solidago ulmifolia Muhl. ex Willd.

* Symphyotrichum patens (Aiton) G.L. Nesom var. patens (=Aster patens Aiton)

* Symphyotrichum subulatum (Michx) G.L. Nesom (=Aster subulatus Michx.)

$\sim$ Verbesina helianthoides Michx.

* Vernonia baldwinii Torr.

Balsaminaceae - Touch-Me-Not Family Impatiens capensis Meerb.

Berberidaceae - Barberry Family

Podophyllum peltatum $\mathrm{L}$.

Bignoniaceae - Trumpet Creeper Family

Campsis radicans (L.) Seem. ex Bureau

Boraginaceae - Borage Family

$\sim$ Lithospermum caroliniense (Walter ex J.F. Gmel.) MacMill. Myosotis verna Nutt.

Brassicaceae - Mustard Family

Cardamine pensylvanica Muhl. ex Willd.

$\sim$ Lepidium virginicum $\mathrm{L}$.

Buddlejaceae - Butterfly-Bush Family

Polypremum procumbens $\mathrm{L}$.

Cabombaceae - Water Shield Family

Brasenia schreberi J. F. Gmel.

Callitrichaceae - Water-Starwort Family

Callitriche heterophylla Pursh

Campanulaceae - Bellflower Family

$\sim$ Triodanis perfoliata (L.) Nieuwl.

Caprifoliaceae - Honeysuckle Family

Lonicera japonica Thunb.

Symphoricarpos orbiculatus Moench

Viburnum rufidulum Raf. common prairie goldenrod

Missouri goldenrod

fragrant goldenrod

rough-leaved goldenrod

elmleaf goldenrod

late purple aster

salt marsh aster

yellow crownbeard

western ironweed

spotted touch-me-not

May apple

trumpet creeper

plains pucoon

early scorpiongrass

bitter cress

poorman's peppergrass

juniperleaf

water shield

water-starwort

clasping Venus looking-glass

Japanese honeysuckle

buckbrush

rusty blackhaw

Clark, L.G. 
Caryophyllaceae - Pink Family

Arenaria serpyllifolia L.

Paronychia drummondii Torr. \& A. Gray

Stellaria media (L.) Vill.

Ceratophyllaceae - Hornwort Family

Ceratophyllum demersum $\mathrm{L}$.

Cistaceae - Rockrose Family

Lechea villosa Elliott

Clusiaceae - Mangosteen Family

Hypericum drummondii (Grev. \& Hook.) Torr. \& A. Gray

$\sim$ Hypericum hypericoides (L.) Crantz

$\sim$ Hypericum prolificum L. (=H. spathulatum (Spach.) Steud.

Convolvulaceae - Morning Glory Family

Ipomoea pandurata (L.) G. Mey.

$\sim$ Stylisma pickeringii (Torr. ex M.A. Curtis) A. Gray

Cornaceae - Dogwood Family

Cornus florida $\mathrm{L}$.

Nyssa sylvatica Marsh.

Droseraceae - Sundew Family

$\sim$ Drosera brevifolia Pursh

Ericaceae - Heath Family

$\sim$ Vaccinium arboreum Marsh.

Euphorbiaceae - Spurge Family

Acalypha rhomboidea Raf.

Acalypha virginica $\mathrm{L}$.

Chamaesyce serpens (Kunth) Small (=Euphorbia serpens Kunth)

Cnidoscolus texanus (Müll. Arg.) Small

Croton capitatus Michx.

Croton glandulosus $\mathrm{L}$.

Croton willdenowii G.L. Webster (=Crotonopsis elliptica Willd.)

Stillingia sylvatica L.

Fabaceae - Pea Family

Apios americana Medik.

Astragalus distortus Torr. \& A. Gray

* Baptisia bracteata Muhl. ex Elliott

Baptisia leucophaea Nutt. var. leucophaea (Nutt.)

Kartesz \& Gandhi (=B. leucophaea Nutt.)

Cercis canadensis $\mathrm{L}$. thyme-leaved sandwort

Drummond's nailwort

chickweed

coontail

pinweed

nits-and-lice

St. Andrew's cross

St. John's wort

wild potatovine

stylisma

flowering dogwood

black gum

sundew

farkleberry

rhombic copperleaf

three seeded Mercury

round-leaved spurge

Texas bullnettle

woolly croton

sand croton

rush-foil

queen's delight

ground nut

bentpod milkvetch

plains wild indigo

white wild indigo

redbud

Clark, L. G. 
Chamaecrista fasciculata (Michx.) Greene var. fasciculata (=Cassia fasciculata Michx.)

Chamaecrista nictitans (L.) Moench ssp. nictitans var. nictitans (=Cassia nictitans $L$.)

Clitoria mariana $\mathrm{L}$.

Dalea phleoides (Torr. \& A. Gray) Shinners var. phleoides (=Petalostemon phleoides Torr. \& A. Gray)

Desmodium paniculatum (L.) DC. var. paniculatum

Desmodium sessilifolium (Torr.) Torr. \& A. Gray

Desmodium viridiflorum (L.) DC.

Galactia regularis (L.) Britton, Sterns \& Poggenb.

Gleditsia triacanthos L. (=Caesalpiniaceae Family)

Lespedeza stuevei Nutt.

Mimosa nutallii (DC. ex Britton \& Rose) B.L. Turner

(=Schrankia nuttallii (DC. ex Britton \& Rose) Standl.)

Mimosa microphylla Dryand. (=Schrankia ucinata Willd.)

Orbexilum pendunculatum (Mill.) Rydb. var. psoralioides (Walter) Isely (=Psoralea psoraloides (Walt.) Cory)

* Orbexilum simplex (Nutt. ex Torr. \& A. Gray) Rydb. (=Psoralea simplex (Nutt. ex Torr. \& A. Gray) Rydb.)

Pediomelum digitatum (Nutt. ex Torr. \& A. Gray) Isely (=Psoralea digitata Nutt. ex Torr. \& A. Gray)

* Pediomelum hypogaeum (Nutt. ex Torr. \& A. Gray) Rydb. var. subulatum (Bush) J. Grimes (=Psoralea subulata Bush) Rhynchosia latifolia Nutt. ex Torr. \& A. Gray

Strophostyles helvola (L.) Elliott

Stylosanthes biflora (L.) Britton, Sterns \& Poggenb.

$\sim$ Tephrosia virginiana (L.) Pers.

Trifolium campestre Schreb.

Vicia sativa $\mathrm{L}$.

Fagaceae - Beech Family

Quercus falcata Michx.

Quercus falcata Michx

$(=Q$. falcata Michx. var. triloba (Michx.) Nutt)

* Quercus incana Bartram

Quercus nigra L.

* Quercus phellos L.

Quercus stellata Wangenh.

Quercus velutina Lam.

Fumariaceae - Fumitory Family

Corydalis micrantha (Engelm. ex A. Gray) A. Gray

Geraniaceae - Geranium Family

Geranium carolinianum L. partridge pea

sensitive pea

butterfly pea

longbract prairie clover

tall tickclover

sessile-leaved tickclover

velvetleaf tickclover

downey milkpea

honey locust

tall lespedeza

sensitive briar

catclaw briar

Sampson's snakeroot

singlestem scurf pea

palm-leaved scurf pea

Sara scurf pea

broadleaf snoutbean

wild bean

pencil-flower

goat's rue

low hop clover

common vetch

southern red oak

southern red oak

bluejack oak

water oak

willow oak

post oak

black oak

slender fumewort

Carolina cranesbill

Clark, L.G. 
Hydrophyllaceae - Waterleaf Family

* Hydrolea ovata Nutt. ex Choisy

Phacelia strictiflora (Engelm. \& A. Gray) A. Gray var. robbinsii Constance

Juglandaceae - Walnut Family

Carya myristiciformis (Michx. f.) Nutt.

Carya texana Buckley

Lamiaceae - Mint Family

* Lycopus virginicus $L$.

Monarda punctata L. Monarda russeliana Nutt. ex Sims.

$\sim$ Prunella vulgaris $\mathrm{L}$.

$\sim$ Pycnanthemum albescens Torr. \& A. Gray

* Pycnanthemum tenuifolium Schrad.

Salvia lyrata L. Scutellaria elliptica Muhl. ex Spreng.

* Scutellaria laterifolia L.

Scutellaria parvula Michx. var. missouriensis (Torr.)

Goodman \& C.A. Lawson

(=S. parvula Michx. var. leonardii (Epling) Fernald)

Lauraceae - Laurel Family

Sassafras albidum (Nutt.) Nees

(=S. albidium (Nutt.) Nees var. molle (Raf.) Fernald

Lentibulariaceae - Bladderwort Family

Utricularia gibba L. (=U. biflora Lam.)

Lythraceae - Loosestrife Family

Rotala ramosior (L.) Koehne

Melastomaceae - Melastome Family

Rhexia mariana L.

Menyanthaceae - Buckbean Famiy

Nymphoides peltata (S.G. Gmel.) Kuntze

Monotropaceae - Indian Pipe Family

Monotropa hypopithys L.

Nymphaeaceae - Water Lily Family

Nuphar lutea (L.) Sm.

Nymphaea odorata Aiton hairy hydrolea

prairie blue curls

nutmeg hickory

black hickory

Virginia bugleweed

horsemint

red spotted horsemint

heal-all

whiteleaf mountainmint

narrowleaf mountainmint

lyreleaf age

hairy skullcap

sideflowering skullcap

skullcap

sassafras

twoflower bladderwort

toothcup

meadow beauty

yellow floating heart

pinesap

yellow pond lily

American water lily

Clark, L. G. 
Onagraceae - Evening Primrose Family

Ludwigia alternifolia L.

Oenothera laciniata Hill

Oxalidaceae - Wood Sorrel Family

Oxalis stricta $\mathrm{L}$.

Oxalis violaceae $\mathrm{L}$.

Plantaginaceae - Plantain Family

Plantago lanceolata L.

Plantago patagonica Jacq.

Plantago virginica $\mathrm{L}$.

Plantago wrightiana Decne.

Polemoniaceae - Phlox Family

Phlox glaberrima L.

Phlox pilosa $\mathrm{L}$.

Polygalaceae - Milkwort Family

* Polygala sanguinea L.

Polygonaceae - Buckwheat Family

$\sim$ Eriogonum longifolium Nutt.

* Eriogonum multiflorum Benth.

$\sim$ Polygonella americana (Fisch. \& C.A. Mey.) Small

$\sim$ Polygonum hydropiperoides Michx.

Polygonum persicaria L.

* Polygonum sagittatum $\mathrm{L}$.

$\sim$ Rumex hastatulus Baldw.

Primulaceae - Primrose Family

Hottonia inflata Elliott

* Lysimachia lanceolata Walter

Ranunculaceae - Buttercup Family

Delphinium carolinianum Walter

Ranunculus laxicaulis (Torr. \& A. Gray) Darby

Rhamnaceae - Buckthorn Family

Berchemia scandens (Hill.) K. Koch

Ceanothus americanus $\mathrm{L}$.

Frangula caroliniana (Walter) A. Gray

(=Rhamnus caroliniana Walter)

Rosaceae - Rose Family

Crataegus spathulata Michx.

Potentilla simplex Michx. bushy seedbox

cutleaf evening primrose

yellow wood sorrel

violet wood sorrel

buckhorn plantain

wooly plantain

paleseed plantain

Wright's plantain

smooth phlox

prairie phlox

blood polygala

longleaf eriogonum

heartsepal wild buckwheat

southern jointweed

mild water pepper

lady's thumb

arrowvine

heartwing sorrel

American featherfoil

lanceleaf loosestrife

prairie larkspur

spearwort

rattan vine

New Jersey tea

buckthorn

littlehip hawthorn

old-field cinquefoil

Clark, L.G. 
Prunus serotina Ehrh.

Rubus occidentalis $\mathrm{L}$.

Rubus ostryifolius Rydb.

Rubiaceae - Madder Family

Cephalanthus occidentalis $\mathrm{L}$.

Diodia teres Walter

$\sim$ Galium arkansanum A. Gray

Galium circaezans Michx.

$\sim$ Galium obtusum Bigelow

Galium pilosum Aiton

Houstonia micrantha (Shinners) Terrell

(=Hedyotis australis W.H. Lewis \& D.M. Moore)

Sapotaceae - Sapodilla Family

Sideroxylon lanuginosum Michx. ssp. lanuginosum (=Bumelia lanuginosa (Michx.) Pers.)

Scrophulariaceae - Figwort family

* Agalinis tenuifolia (Vahl.) Raf.

* Castilleja coccinea (L.) Spreng.

Castilleja indivisa Engelm.

Collinsia violacea Nutt.

Gratiola virginiana L.

Nuttallanthus canadensis (L.) D.A. Sutton (=Linaria canadensis (L.) Chaz.)

Lindernia dubia (L.) Pennell

Pedicularis canadensis L. ssp. canadensis

(=P. canadensis L. var. dobbsii Fernald)

* Penstemon laxiflorus Pennell

Penstemon murrayanus Hook.

Solanaceae - Potato Family

Physalis heterophylla Nees.

Solanum carolinense L.

Ulmaceae - Elm Family

Celtis tenuifolia Nutt.

Urticaceae - Nettle Family

$\sim$ Boehmeria cylindrica (L.) Sw.

Valerianaceae - Valerian Family

$\sim$ Valerianella radiata (L.) Dufr.

Verbenaceae - Verbena Family

Callicarpa americana L. black cherry

blackberry

highbush blackberry

buttonbush

rough buttonweed

Arkansas bedstraw

woods bedstraw

bluntleaf bedstraw

hairy bedstraw

bluet

chittamwood

slenderleaf agalinus

Indian paintbrush

Indian paintbrush

violet collinsia

Virginia hedgehyssop

blue toadflax

yellowseed false pimpernell

common lousewort

loose flower penstemon

cupleaf penstemon

clammy ground cherry

Carolina horsenettle

dwarf hackberry

false nettle

common beaked cornsalad

American beautyberry

Clark, L. G. 
Phryma leptostachya L.

* Verbena simplex Lehm.

Violaceae - Violet Family

Viola villosa Walter

\section{Vitaceae - Grape Family}

Ampelopsis arborea (L.) Koehne

Parthenocissus quinquefolia (L.) Planch.

Vitis aestivalis Michx.

Vitis rotundifolia Michx. lopseed

narrow-leaved verbena

wooly violet

peppervine

Virginia creeper

pigeon grape

muscadine

Clark, L.G. 